\title{
Nontrivial solutions for discrete Kirchhoff-type problems with resonance via critical groups
}

Jinping Yang and Jinsheng Liu*

"Correspondence: I_j_s@163.com College of Mathematics, Taiyuan University of Technology, Taiyuan, Shanxi 030024, P.R. China

\begin{abstract}
In this paper, we study the existence of nontrivial solutions for discrete Kirchhoff-type problems with resonance at both zero and infinity by using variational methods and the computations of critical groups.
\end{abstract}

Keywords: discrete Kirchhoff-type problem; variational method; critical point; critical group

\section{Introduction and main results}

Let $\mathbb{R}, \mathbb{Z}, \mathbb{N}$ be the sets of all real numbers, integers and natural numbers, respectively. In this paper, we consider the existence of nontrivial solutions for the following discrete Kirchhoff-type nonlocal problem with Dirichlet boundary condition:

$$
\left\{\begin{array}{l}
-\left(a+b \sum_{k=1}^{N+1}|\Delta u(k-1)|^{2}\right) \Delta^{2} u(k-1)=f(k, u(k)), \quad k \in \mathbb{Z}[1, N] \\
u(0)=u(N+1)=0,
\end{array}\right.
$$

where $N \geq 3$ is a fixed positive integer, $a, b>0$ are real constants, $\mathbb{Z}[1, N]$ denotes the discrete interval $\{1,2, \ldots, N\}$. As usual, $\Delta$ denotes the forward difference operator defined by $\Delta u(k)=u(k+1)-u(k), \Delta^{2} u(k)=\Delta(\Delta u(k))$, and for all $k \in \mathbb{Z}[1, N], f(k, \cdot) \in C^{1}(\mathbb{R}, \mathbb{R})$ satisfies $f(k, 0)=0$. Obviously, problem (1.1) has the trivial solution $u=0$. Hence, we are interested in finding nontrivial solutions of problem (1.1). The existence of nontrivial solutions for problem (1.1) depends on the local properties of $f$ near zero and near infinity. In this work, we consider the cases where $f$ satisfies the asymptotic conditions

$$
\lim _{|t| \rightarrow 0} \frac{f(k, t)}{a t}=f_{0}
$$

and

$$
\lim _{|t| \rightarrow \infty} \frac{f(k, t)}{b t^{3}}=f_{\infty}
$$

for all $k \in \mathbb{Z}[1, N]$.

@2013 Yang and Liu; licensee Springer. This is an Open Access article distributed under the terms of the Creative Commons Attribution License (http://creativecommons.org/licenses/by/2.0), which permits unrestricted use, distribution, and reproduction in any medium, provided the original work is properly cited. 
Let $\lambda_{i}, i \in \mathbb{Z}[1, N]$ be the eigenvalues of the linear eigenvalue problem

$$
\left\{\begin{array}{l}
-\Delta^{2} u(k-1)=\lambda u(k), \quad k \in \mathbb{Z}[1, N] \\
u(0)=u(N+1)=0
\end{array}\right.
$$

and let $\xi_{i}=\left(\xi_{i}(1), \xi_{i}(2), \ldots, \xi_{i}(N)\right)^{T}$ be an eigenvector corresponding to the eigenvalue $\lambda_{i}$, then $\left\{\xi_{1}, \xi_{2}, \ldots, \xi_{N}\right\}$ is an orthogonal basis of $\mathbb{R}^{N}$, where $\lambda_{i}=4 \sin ^{2} \frac{i \pi}{2(N+1)}, \xi_{i}(j)=\sin \frac{i j \pi}{N+1}$, $i, j \in \mathbb{Z}[1, N]$.

Denote by $\mu_{1}, \mu_{2}$ the minimum eigenvalue and the maximum eigenvalue of the nonlinear eigenvalue problem, respectively,

$$
\left\{\begin{array}{l}
-\left(\sum_{k=1}^{N+1}|\Delta u(k-1)|^{2}\right) \Delta^{2} u(k-1)=\mu u^{3}(k), \quad k \in \mathbb{Z}[1, N] \\
u(0)=u(N+1)=0
\end{array}\right.
$$

(see Theorem 2.1).

Remark 1.1 By referring to the notion of resonance of elliptic equations of Kirchhofftype $[1,2]$, we call problem (1.1) resonant at zero if there exists some $m \in \mathbb{Z}[1, N]$ such that $f_{0}=\lambda_{m}$ and resonant at infinity if there exists some eigenvalue $\mu$ of problem (1.4) such that $f_{\infty}=\mu$.

To state our main results, we define

$$
\begin{aligned}
& F(k, t)=\int_{0}^{t} f(k, s) d s, \quad k \in \mathbb{Z}[1, N], t \in \mathbb{R}, \\
& \mu_{0}=\sharp\left\{\lambda_{i} \mid \lambda_{i}<f_{0}, i \in \mathbb{Z}[1, N]\right\}
\end{aligned}
$$

and make the following assumptions:

$\left(\mathrm{F}_{0}\right) \quad$ There exists $\delta_{1}>0$ such that $F(k, t) \geq \frac{a}{2} \lambda_{m} t^{2}+\frac{b}{4} m \lambda_{m}^{2} t^{4}, k \in \mathbb{Z}[1, N],|t| \leq \delta_{1}$.

$\left(\mathrm{F}_{\infty}^{1}\right) \lim _{|t| \rightarrow \infty}(t f(k, t)-4 F(k, t))=+\infty, k \in \mathbb{Z}[1, N]$.

$\left(\mathrm{F}_{\infty}^{2}\right)$ There exist $\delta_{2}>0$ and $c \in\left(\frac{a}{2} \lambda_{N},+\infty\right)$ such that $F(k, t) \geq c t^{2}+\frac{b}{4} f_{\infty} t^{4}, k \in \mathbb{Z}[1, N]$, $|t| \geq \delta_{2}$.

Firstly, we consider the case that problem (1.1) is only resonant at zero.

Theorem 1.1 If $f_{0}=\lambda_{m}$ and $\left(\mathrm{F}_{0}\right)$ hold, then problem (1.1) has at least one nontrivial solution in each of the following cases:

(i) $f_{\infty}<\mu_{1}$;

(ii) $f_{\infty}>\mu_{2}$ and $m \neq N$.

Next, we investigate the case that problem (1.1) is resonant at both zero and infinity.

Theorem 1.2 If $f_{0}=\lambda_{m}$ and $\left(\mathrm{F}_{0}\right)$ hold, then problem (1.1) has at least one nontrivial solution in each of the following cases:

(i) $\left(\mathrm{F}_{\infty}^{1}\right)$ and $f_{\infty}=\mu_{1}$;

(ii) $\left(\mathrm{F}_{\infty}^{2}\right), f_{\infty}=\mu_{2}$ and $m \neq N$. 
Thirdly, we study the case that problem (1.1) is only resonant at infinity.

Theorem 1.3 If $f_{0} \neq \lambda_{i}, i \in \mathbb{Z}[1, N]$ hold, then problem (1.1) has at least one nontrivial solution in each of the following cases:

(i) $\left(\mathrm{F}_{\infty}^{1}\right), f_{\infty}=\mu_{1}$ and $\mu_{0} \neq 0$;

(ii) $\left(\mathrm{F}_{\infty}^{2}\right), f_{\infty}=\mu_{2}$ and $\mu_{0} \neq N$.

Finally, we deal with the case that problem (1.1) is non-resonant.

Theorem 1.4 If $f_{0} \neq \lambda_{i}, i \in \mathbb{Z}[1, N]$ hold, then problem (1.1) has at least one nontrivial solution in each of the following cases:

(i) $f_{\infty}<\mu_{1}$ and $\mu_{0} \neq 0$;

(ii) $f_{\infty}>\mu_{2}$ and $\mu_{0} \neq N$.

Now, we give some examples to illustrate our main results.

\section{Example 1.1 If}

$$
f(k, t)= \begin{cases}a \lambda_{1} t+b \lambda_{1}^{2} t^{3}, & |t| \leq 1, \\ f_{1}(t), & 1<|t|<10, \\ \frac{1}{2} b \mu_{1} t^{3}, & |t| \geq 10,\end{cases}
$$

where $f_{1}$ is a smooth connection such that $f \in C^{1}(\mathbb{Z}[1, N] \times \mathbb{R}, \mathbb{R})$, it is easy to know that $f_{0}=\lambda_{1}, f_{\infty}=\frac{1}{2} \mu_{1}<\mu_{1}$ and the condition $\left(\mathrm{F}_{0}\right)$ holds, so problem (1.1) has at least a nontrivial solution by (i) of Theorem 1.1.

Example 1.2 If

$$
f(k, t)= \begin{cases}a \lambda_{1} t+b \lambda_{1}^{2} t^{3}, & |t| \leq 1 \\ f_{2}(t), & 1<|t|<10 \\ 2 b \mu_{2} t^{3}, & |t| \geq 10\end{cases}
$$

where $f_{2}$ is a smooth connection such that $f \in C^{1}(\mathbb{Z}[1, N] \times \mathbb{R}, \mathbb{R})$, it is easy to know that $f_{0}=\lambda_{1}, f_{\infty}=2 \mu_{2}>\mu_{1}, N \neq 1$ and the condition $\left(\mathrm{F}_{0}\right)$ holds, so problem (1.1) has at least a nontrivial solution by (ii) of Theorem 1.1.

It is well known that in different fields of research, such as computer science, mechanical engineering, control systems, artificial or biological neural networks, economics and many others, the mathematical modeling of important questions leads naturally to the consideration of nonlinear difference equations. For this reason, in recent years, resonant difference problems via variational methods have been widely studied by various authors (see [3-12] and the references therein). For example, in [4-7, 10, 12], the authors considered the following second-order difference boundary value problem:

$$
\left\{\begin{array}{l}
-\Delta^{2} u(k-1)=f(k, u(k)), \quad k \in \mathbb{Z}[1, N] \\
u(0)=u(N+1)=0,
\end{array}\right.
$$


where $f$ satisfies the resonance condition

$$
\lim _{|t| \rightarrow 0} \frac{f(k, t)}{t}=\lambda_{m}
$$

or

$$
\lim _{|t| \rightarrow \infty} \frac{f(k, t)}{t}=\lambda_{l}
$$

for all $k \in \mathbb{Z}[1, N]$. They investigated the existence and multiplicity of nontrivial solutions for problem (1.6) by using various methods and techniques, such as minimax methods, bifurcation theory, critical groups, Morse theory and so on. However, to our knowledge, there are few results on the existence of nontrivial solutions of a discrete Kirchhoff-type resonance problem which is an extension of problem (1.6).

In addition, as is well known, the equation

$$
-\left(a+b \int_{0}^{l} u_{x}^{2} d x\right) u_{x x}=f(x, u), \quad 0<x<l
$$

was the stationary case of the equation

$$
u_{t t}-\left(a+b \int_{0}^{l} u_{x}^{2} d x\right) u_{x x}=f(x, u), \quad 0<x<l, t>0
$$

proposed by Kirchhoff [13] as an extension of the classical D'Alembert wave equation for free vibrations of elastic stings. After the famous article by Lions [14], this type of problems has been the subject of numerous studies. Due to the importance of equation (1.6), in recent years, many authors have studied the existence of solutions of equation (1.6) and the corresponding general elliptic equations with Dirichlet boundary value condition (see, e.g., $[1,2,15-18]$ and the references therein). Obviously, problem (1.1) is the discrete form of equation (1.6). However, there are significant differences between (1.1) and (1.6) in some aspects such as properties of eigenvalues (see Theorem 2.1 and Proposition 3.2 in [1]), which justifies the necessity of research on problem (1.1).

In the current paper, we conclude the existence of eigenvalues for nonlinear eigenvalue problem (1.4) via the Lagrange multiplier rule. This appears to be first such result for eigenvalue problem (1.4). Furthermore, the existence of nontrivial solutions of discrete Kirchhoff-type problem (1.1) with resonance at both zero and infinity is also studied by employing the critical point theory, especially the local linking, Morse theory and the computations of critical groups.

The rest of this paper is organized as follows. In Section 2, we give the energy functional of problem (1.1) and study the eigenvalue of problem (1.4). In order to prove our main results, some facts about the critical groups are also recalled in this section. In Section 3, the proofs of main results are provided. 


\section{Preliminaries}

Let

$$
\begin{aligned}
& A=\left(\begin{array}{cccccc}
2 & -1 & 0 & \cdots & 0 & 0 \\
-1 & 2 & -1 & \cdots & 0 & 0 \\
0 & -1 & 2 & \cdots & 0 & 0 \\
\vdots & \vdots & \vdots & & \vdots & \vdots \\
0 & 0 & 0 & \cdots & 2 & -1 \\
0 & 0 & 0 & \cdots & -1 & 2
\end{array}\right)_{N \times N}, \\
& u=(u(1), u(2), \ldots, u(N))^{T}, \\
& f(u)=(f(1, u(1)), f(2, u(2)), \ldots, f(N, u(N)))^{T}, \\
& f^{\prime}(u)=\operatorname{diag}\left(f_{t}^{\prime}(1, u(1)), f_{t}^{\prime}(2, u(2)), \ldots, f_{t}^{\prime}(N, u(N))\right),
\end{aligned}
$$

and let $H=\mathbb{R}^{N}$ be an $N$-dimensional real Hilbert space with the inner product $\langle u, v\rangle=$ $u^{T} A v$ and the norm $\|u\|=\sqrt{\langle u, u\rangle}$. For $1 \leq p<+\infty$, we denote $\|u\|_{p}=\left(\sum_{k=1}^{N}|u(k)|^{p}\right)^{\frac{1}{p}}$. Then, for all $u \in H$, we have

$$
\lambda_{1}\|u\|_{2}^{2} \leq\|u\|^{2} \leq \lambda_{N}\|u\|_{2}^{2}
$$

and

$$
\|u\|_{4}^{2} \leq\|u\|_{2}^{2} \leq \sqrt{N}\|u\|_{4}^{2} .
$$

Obviously, problem (1.1) can be expressed as the following matrix form:

$$
\left(a+b\|u\|^{2}\right) A u=f(u)
$$

which motivates us to consider

$$
J(u)=\frac{a}{2}\|u\|^{2}+\frac{b}{4}\|u\|^{4}-\sum_{k=1}^{N} F(k, u(k)), \quad u \in H .
$$

Noting that $f(k, \cdot) \in C^{1}(\mathbb{R}, \mathbb{R}), J$ is a functional with Fréchet derivatives given by

$$
\begin{aligned}
& J^{\prime}(u)=\left(a+b\|u\|^{2}\right) u-A^{-1} f(u), \quad u \in H, \\
& J^{\prime \prime}(u)=\left(a+b\|u\|^{2}\right) I_{N}+2 b u(A u)^{T}-A^{-1} f^{\prime}(u), \quad u \in H .
\end{aligned}
$$

Hence the solutions of problem (1.1) are exactly the critical points of $J$ in $H$ and $J \in$ $C^{2}(H, \mathbb{R})$.

Now we consider nonlinear eigenvalue problem (1.4). Firstly, we introduce the Lagrange multiplier rule.

Lemma 2.1 [19] Let $X, Y$ be real Banach spaces, $B_{r}\left(x_{0}\right) \subset X, \varphi: B_{r}\left(x_{0}\right) \rightarrow \mathbb{R}$ and $F$ : $B_{r}\left(x_{0}\right) \rightarrow Y$ continuously differentiable, $F\left(x_{0}\right)=0$ and $R\left(F^{\prime}\left(x_{0}\right)\right)$ closed. Suppose also that 
$\varphi\left(x_{0}\right)=\min \left\{\varphi(x): x \in B_{r}\left(x_{0}\right)\right.$ and $\left.F(x)=0\right\}$. Then there exist Lagrange multipliers $\lambda \in \mathbb{R}$ and $y^{*} \in Y^{*}$, not all zero, such that $\lambda \varphi\left(x_{0}\right)+\left(F^{\prime}\left(x_{0}\right)\right)^{*} y^{*}=0$. If $R\left(F^{\prime}\left(x_{0}\right)\right)=Y$, then $\lambda \neq 0$.

Theorem 2.1 Problem (1.4) only has finitely many eigenvalues which are in $\left[\lambda_{1}^{2}, N \lambda_{N}^{2}\right]$, and moreover,

$$
\mu_{1}\|u\|_{4}^{4} \leq\|u\|^{4} \leq \mu_{2}\|u\|_{4}^{4}, \quad u \in H
$$

Proof Firstly, we prove the existence of $\mu_{1}$ and $\mu_{2}$.

Indeed, for any $u=(u(1), u(2), \ldots, u(N))^{T} \in \mathbb{R}^{N}$, denote

$$
u^{3}=\left(u^{3}(1), u^{3}(2), \ldots, u^{3}(N)\right)^{T}
$$

then problem (1.4) can be written as the following matrix form:

$$
\left(u^{T} A u\right) A u=\mu u^{3}
$$

and the energy functional corresponding to problem (1.4) can be expressed as

$$
\phi(u)=\frac{1}{4}\left(u^{T} A u\right)^{2}-\frac{\mu}{4} \sum_{k=1}^{N} u^{4}(k)=\frac{1}{4}\|u\|^{4}-\frac{\mu}{4}\|u\|_{4}^{4} .
$$

By a simple computation, we obtain

$$
\phi^{\prime}(u)=\|u\|^{2} u-\mu A^{-1} u^{3} .
$$

If $u$ is a nonzero solution of problem (1.4) corresponding to some eigenvalue $\mu$, then

$$
\left\langle\phi^{\prime}(u), u\right\rangle=\|u\|^{4}-\mu\|u\|_{4}^{4}=0 \text {. }
$$

Therefore we can get $\mu=\frac{\|u\|^{4}}{\|u\|_{4}^{4}}$. It follows from (2.1) and (2.2) that

$$
\mu \in\left[\lambda_{1}^{2}, N \lambda_{N}^{2}\right]
$$

Consider the following extreme value problem:

$$
I(u)=\|u\|^{4}, \quad u \in S:=\left\{u \in H: \varphi(u)=\|u\|_{4}^{4}=1\right\} .
$$

Noting that $I(u)$ is continuous on $\|u\|_{4}^{4}=1$, which is a bounded closed set in $\mathbb{R}^{N}$, there exist $u_{1} \neq 0$ and $u_{2} \neq 0$ on the sphere $\|u\|_{4}^{4}=1$ such that

$$
I\left(u_{1}\right)=\min _{\|u\|_{4}^{4}=1} I(u)
$$

and

$$
I\left(u_{2}\right)=\max _{\|u\|_{4}^{4}=1} I(u)
$$


By Lemma 2.1, there exist $\mu_{1}, \mu_{2} \in\left[\lambda_{1}^{2}, N \lambda_{N}^{2}\right]$ such that

$$
I^{\prime}\left(u_{1}\right)=\mu_{1} \varphi^{\prime}\left(u_{1}\right), \quad I^{\prime}\left(u_{2}\right)=\mu_{2} \varphi^{\prime}\left(u_{2}\right)
$$

i.e.,

$$
\phi^{\prime}\left(u_{i}\right)=\left\|u_{i}\right\|^{2} u_{i}-\mu_{i} A^{-1} u_{i}^{3}=0, \quad i=1,2 .
$$

Therefore, for any $N \geq 3$, we can find at least two eigenvalues $\mu_{1}$ and $\mu_{2}$ of problem (1.4) such that

$$
\mu_{1}=\frac{\left\|u_{1}\right\|^{4}}{\left\|u_{1}\right\|_{4}^{4}}=\left\|u_{1}\right\|^{4}=I\left(u_{1}\right)<I\left(u_{2}\right)=\left\|u_{2}\right\|^{4}=\frac{\left\|u_{2}\right\|^{4}}{\left\|u_{2}\right\|_{4}^{4}}=\mu_{2}
$$

If $\mu$ is also an eigenvalue of problem (1.4), then there exists $u \neq 0$ on the sphere $\|u\|_{4}^{4}=1$ such that

$$
\mu_{1}=\left\|u_{1}\right\|^{4}=I\left(u_{1}\right) \leq I(u)=\|u\|^{4}=\mu \leq I\left(u_{2}\right)=\left\|u_{2}\right\|^{4}=\mu_{2}
$$

To sum up, $\mu_{1}$ is the minimum eigenvalue and $\mu_{2}$ is the maximum eigenvalue of problem (1.4). Meanwhile, for any $u \in H$ and $u \neq 0$, setting $v=\frac{u}{\|u\|_{4}}$, we have

$$
\mu_{1}\|u\|_{4}^{4} \leq\|u\|^{4} \leq \mu_{2}\|u\|_{4}^{4}
$$

Now, we claim that the number of eigenvalues of problem (1.4) is finite.

Clearly, for any $c \in \mathbb{R}$ and $c \neq 0$, if $u$ is a solution of the equation $\|u\|^{2} A u=\mu u^{3}$ for some $\mu$, so is $c u$. Thus we can suppose that $\|u\|^{2}=1$. Hence, we only need to consider the following equations:

$$
\left\{\begin{array}{l}
A u=\mu u^{3} \\
\|u\|^{2}=1
\end{array}\right.
$$

By $A u=\mu u^{3}$, we can obtain

$$
\left\{\begin{array}{l}
2 u(1)-u(2)=\mu u^{3}(1), \\
2 u(2)-u(1)-u(3)=\mu u^{3}(2), \\
2 u(3)-u(2)-u(4)=\mu u^{3}(3), \\
\cdots \\
2 u(N-2)-u(N-3)-u(N-1)=\mu u^{3}(N-2), \\
2 u(N-1)-u(N-2)-u(N)=\mu u^{3}(N-1), \\
2 u(N)-u(N-1)=\mu u^{3}(N) .
\end{array}\right.
$$


Obviously, $u(1) \neq 0$ and $\mu \neq 0$. Thus

$$
\left\{\begin{array}{l}
\frac{2 u(2)-u(1)-u(3)}{2 u(1)-u(2)}=\left(\frac{u(2)}{u(1)}\right)^{3}, \\
\frac{2 u(3)-u(2)-u(4)}{2 u(1)-u(2)}=\left(\frac{u(3)}{u(1)}\right)^{3}, \\
\cdots \\
\frac{2 u(N-2)-u(N-3)-u(N-1)}{2 u(1)-u(2)}=\left(\frac{u(N-2)}{u(1)}\right)^{3}, \\
\frac{2 u(N-1)-u(N-2)-u(N)}{2 u(1)-u(2)}=\left(\frac{u(N-1)}{u(1)}\right)^{3}, \\
\frac{2 u(N)-u(N-1)}{2 u(1)-u(2)}=\left(\frac{u(N)}{u(1)}\right)^{3} .
\end{array}\right.
$$

Furthermore, let

$$
\begin{aligned}
& u(2)=c_{2} u(1), \quad u(3)=c_{3} u(1), \quad \ldots, \\
& u(N-1)=c_{N-1} u(1), \quad u(N)=c_{N} u(1),
\end{aligned}
$$

then we easily get

$$
\left\{\begin{array}{l}
\frac{2 c_{2}-1-c_{3}}{2-c_{2}}=c_{2}^{3} \\
\frac{2 c_{3}-c_{2}-c_{4}}{2-c_{2}}=c_{3}^{3} \\
\cdots \\
\frac{2 c_{N-2}-c_{N-3}-c_{N-1}}{2-c_{2}}=c_{N-2}^{3}, \\
\frac{2 c_{N-1}-c_{N-2}-c_{N}}{2-c_{2}}=c_{N-1}^{3} \\
\frac{2 c_{N}-c_{N-1}}{2-c_{2}}=c_{N}^{3}
\end{array}\right.
$$

Consequently,

$$
\left\{\begin{array}{l}
c_{3}=2 c_{2}-1-\left(2-c_{2}\right) c_{2}^{3}:=f_{3}\left(c_{2}\right), \\
c_{4}=2 c_{3}-c_{2}-\left(2-c_{2}\right) c_{3}^{3}:=f_{4}\left(c_{2}\right), \\
\ldots \\
c_{N-1}=2 c_{N-2}-c_{N-3}-\left(2-c_{2}\right) c_{N-2}^{3}:=f_{N-1}\left(c_{2}\right), \\
c_{N}=2 c_{N-1}-c_{N-2}-\left(2-c_{2}\right) c_{N-1}^{3}:=f_{N}\left(c_{2}\right), \\
2 f_{N}\left(c_{2}\right)-f_{N-1}\left(c_{2}\right)=\left(2-c_{2}\right)\left(f_{N}\left(c_{2}\right)\right)^{3} .
\end{array}\right.
$$

Apparently, the last equation in (2.6) is an algebraic equation of finite degree with unknown $c_{2}$. By the fundamental theorem of algebra, this equation only has finitely many solutions in the field of complex numbers. Thus, $c_{3}, c_{4}, \ldots, c_{N}$ can be obtained by the first equation, the second equation, $\cdots$, the $(N-2)$ th equation in $(2.6)$, respectively, after $c_{2}$ is determined. Noting that

$$
\begin{aligned}
1+ & c_{2}^{2}+\cdots+c_{N}^{2}-c_{2}-c_{2} c_{3}-c_{3} c_{4}-\cdots-c_{N-1} c_{N} \\
& =\frac{1}{2}\left[1+\left(1-c_{2}\right)^{2}+\left(c_{2}-c_{3}\right)^{2}+\cdots+\left(c_{N-2}-c_{N-1}\right)^{2}+\left(c_{N-1}-c_{N}\right)^{2}+c_{N}^{2}\right]>0,
\end{aligned}
$$


substituting (2.5) into $\|u\|^{2}=1$ and by $2 u(1)-u(2)=\mu u^{3}(1), u(2)=c_{2} u(1)$, we can infer that

$$
\mu=2\left(2-c_{2}\right)\left(1+c_{2}^{2}+\cdots+c_{N}^{2}-c_{2}-c_{2} c_{3}-c_{3} c_{4}-\cdots-c_{N-1} c_{N}\right)
$$

and

$$
u(1)= \pm \sqrt{2}\left(1+c_{2}^{2}+\cdots+c_{N}^{2}-c_{2}-c_{2} c_{3}-c_{3} c_{4}-\cdots-c_{N-1} c_{N}\right)^{-\frac{1}{2}} \neq 0
$$

Hence the number of eigenvalues of problem (1.4) is finite due to the finiteness of $c_{2}, c_{3}, \ldots, c_{N}$. Besides, we can obtain $u(2), u(3), \ldots, u(N)$ by $(2.5)$. This proof is completed.

In the following we recall some facts about the critical groups and Morse theory; see [20-22] for more details.

Definition 2.1 [20] Let $H$ be a Hilbert space and $J \in C^{1}(H, \mathbb{R})$, let $u_{0}$ be an isolated critical point of $J$ with $J\left(u_{0}\right)=c$, and let $U$ be a neighbourhood of $u_{0}$, containing the unique critical point, the group

$$
C_{q}\left(J, u_{0}\right)=H_{q}\left(J^{c} \cap U, J^{c} \backslash\left\{u_{0}\right\} \cap U\right), \quad q \in \mathbb{Z},
$$

is called the $q$ th critical group of $J$ at $u_{0}$, where $J^{c}=\{u \in H \mid J(u) \leq c\}, H_{q}(A, B)$ denotes the $q$ th singular relative homology group of the topological pair $(A, B)$ with coefficients in a field $\mathbb{F}$.

Definition 2.2 [20] Let $H$ be a Hilbert space and $J \in C^{2}(H, \mathbb{R}), J^{\prime}\left(u_{0}\right)=0$. Assume that $H^{-}$is the supremum of the vector subspaces of $H$ on which $J^{\prime \prime}\left(u_{0}\right)$ is negative definite. The Morse index of $J$ at $u_{0}$ is defined as the dimension of $H^{-}$. The nullity of $J$ at $u_{0}$ is defined as the dimension of $\operatorname{ker} J^{\prime \prime}\left(u_{0}\right) \cdot u_{0}$ is called a non-degenerate critical point of $J$ if $J^{\prime \prime}\left(u_{0}\right)$ has a bounded inverse.

Lemma 2.2 [21,22] Let 0 be an isolated critical point of $J \in C^{2}(H, \mathbb{R})$ with the Morse index $\mu_{0}$ and the nullity $v_{0}$. Assume that $J$ has a local linking at 0 with respect to $H=H^{-} \oplus H^{+}$, $l=\operatorname{dim} H^{-}<\infty$, i.e., there exists $\rho>0$ such that

$$
\begin{aligned}
& J(u) \leq 0, \quad \text { for } u \in H^{-},\|u\| \leq \rho ; \\
& J(u) \geq 0, \quad \text { for } u \in H^{+},\|u\| \leq \rho .
\end{aligned}
$$

Then $C_{q}(J, 0)=\delta_{q, l} \mathbb{F}$, if $l=\mu_{0}$ or $l=\mu_{0}+v_{0}$.

Lemma 2.3 [20] Let $u_{0}$ be an isolated critical point of $J \in C^{2}(H, \mathbb{R})$, then the following statements are true:

(i) If $u_{0}$ is a minimum point of $J$, then $C_{q}\left(J, u_{0}\right)=\delta_{q, 0} \mathbb{F}$;

(ii) If $u_{0}$ is a maximum point of $J$ and $\operatorname{dim} H=l<\infty$, then $C_{q}\left(J, u_{0}\right)=\delta_{q, l} \mathbb{F}$;

(iii) If $u_{0}$ is a non-degenerate critical point of $J$ with the Morse index $\mu_{0}$, then

$$
C_{q}\left(J, u_{0}\right)=\delta_{q, \mu_{0}} \mathbb{F} \text {. }
$$




\section{Proofs of main results}

Now we give the proofs of Theorems 1.1-1.4.

Proof of Theorem 1.1 Since (1.2), $f_{0}=\lambda_{m}$ and $\left(\mathrm{F}_{0}\right)$ hold, for any $\varepsilon>0$, there exists $\rho>0$ such that $\frac{\rho}{\sqrt{\lambda_{1}}}=\delta_{1} \leq \delta$ and

$$
\frac{a}{2} \lambda_{m} t^{2}+\frac{b}{4} m \lambda_{m}^{2} t^{4} \leq F(k, t) \leq \frac{a}{2}\left(\lambda_{m}+\varepsilon\right) t^{2}, \quad|t| \leq \delta
$$

Let $H=H_{0}^{-} \oplus H_{0}^{+}, H_{0}^{-}=\operatorname{span}\left\{\xi_{1}, \xi_{2}, \ldots, \xi_{m}\right\}, H_{0}^{+}=\left(H_{0}^{-}\right)^{\perp}$, then $\operatorname{dim} H_{0}^{-}=m<+\infty$. Therefore, by (2.1) and (2.2), it is easy to know that

$$
\lambda_{1}\|u\|_{2}^{2} \leq\|u\|^{2} \leq \lambda_{m}\|u\|_{2}^{2} \leq \sqrt{m} \lambda_{m}\|u\|_{4}^{2}, \quad u \in H_{0}^{-}
$$

and

$$
\lambda_{m+1}\|u\|_{4}^{2} \leq \lambda_{m+1}\|u\|_{2}^{2} \leq\|u\|^{2} \leq \lambda_{N}\|u\|_{2}^{2}, \quad u \in H_{0}^{+} .
$$

For any $u \in H_{0}^{+},\|u\| \leq \rho$ implies that $|u(k)| \leq \delta$. Then we can deduce from (3.1) that

$$
\begin{aligned}
J(u) & =\frac{a}{2}\|u\|^{2}+\frac{b}{4}\|u\|^{4}-\sum_{k=1}^{N} F(k, u(k)) \\
& \geq \frac{a}{2}\|u\|^{2}-\sum_{k=1}^{N} F(k, u(k)) \\
& \geq \frac{a}{2}\|u\|^{2}-\frac{a}{2}\left(\lambda_{m}+\varepsilon\right) \sum_{k=1}^{N} u^{2}(k) \\
& \geq \frac{a}{2}\left(1-\frac{\lambda_{m}+\varepsilon}{\lambda_{m+1}}\right)\|u\|^{2} .
\end{aligned}
$$

Noting that $\lambda_{m}<\lambda_{m+1}$, we can choose $\varepsilon$ small enough such that $J(u) \geq 0$ for $\|u\| \leq \rho$ and $u \in H_{0}^{+}$.

For any $u \in H_{0}^{-},\|u\| \leq \rho$ implies that $|u(k)| \leq \delta$. Thus we see from (3.1) that

$$
\begin{aligned}
J(u) & =\frac{a}{2}\|u\|^{2}+\frac{b}{4}\|u\|^{4}-\sum_{k=1}^{N} F(k, u(k)) \\
& \leq \frac{a}{2}\|u\|^{2}+\frac{b}{4}\|u\|^{4}-\sum_{k=1}^{N}\left(\frac{a}{2} \lambda_{m} u^{2}(k)+\frac{b}{4} m \lambda_{m}^{2} u^{4}(k)\right) \\
& \leq \frac{a}{2}\|u\|^{2}+\frac{b}{4}\|u\|^{4}-\frac{a}{2}\|u\|^{2}-\frac{b}{4} m \lambda_{m}^{2}\|u\|_{4}^{4} \\
& \leq \frac{b}{4} m \lambda_{m}^{2}\|u\|_{4}^{4}-\frac{b}{4} m \lambda_{m}^{2}\|u\|_{4}^{4}=0
\end{aligned}
$$

So, $J$ has a local linking at 0 with respect to $H=H_{0}^{-} \oplus H_{0}^{+}$. Using Lemma 2.2, we can obtain

$$
C_{q}(J, 0)=\delta_{q, m} \mathbb{F}
$$


(i) By (1.3), $f_{\infty}<\mu_{1}$ and the continuity of $F$, we know that for any $\varepsilon>0$, there exists $M_{\varepsilon}>0$ such that $F(k, t) \leq \frac{1}{4} b\left(\mu_{1}-\varepsilon\right) t^{4}+M_{\varepsilon}$ for all $t \in \mathbb{R}$ and $k \in \mathbb{Z}[1, N]$. Together with (2.4), we have

$$
\begin{aligned}
J(u) & =\frac{a}{2}\|u\|^{2}+\frac{b}{4}\|u\|^{4}-\sum_{k=1}^{N} F(k, u(k)) \\
& \geq \frac{a}{2}\|u\|^{2}+\frac{b}{4}\|u\|^{4}-\sum_{k=1}^{N}\left(\frac{1}{4} b\left(\mu_{1}-\varepsilon\right) u^{4}(k)+M_{\varepsilon}\right) \\
& =\frac{a}{2}\|u\|^{2}+\frac{b}{4}\|u\|^{4}-\frac{1}{4} b\left(\mu_{1}-\varepsilon\right)\|u\|_{4}^{4}-N M_{\varepsilon} \\
& \geq \frac{a}{2}\|u\|^{2}-N M_{\varepsilon} .
\end{aligned}
$$

It follows that

$$
J(u) \rightarrow \infty, \quad \text { as }\|u\| \rightarrow \infty,
$$

which implies that $J$ is coercive in $H$. By the continuity of $J, J$ must have a minimum point $u_{1}$. According to Lemma 2.3(i), we conclude that

$$
C_{q}\left(J, u_{1}\right)=\delta_{q, 0} \mathbb{F}
$$

which, together with (3.2) and $m \neq 0$, shows that $u_{1} \neq 0$. So, $J$ has at least a nontrivial critical point.

(ii) It follows from (1.3), $f_{\infty}>\mu_{2}$ and the continuity of $F$ that we can find $\varepsilon>0, M_{\varepsilon}>0$ such that $F(k, t) \geq \frac{b}{4}\left(\mu_{2}+\varepsilon\right) t^{4}-M_{\varepsilon}$ for all $t \in \mathbb{R}$ and $k \in \mathbb{Z}[1, N]$. By (2.1), (2.2) and (2.4), we can see that

$$
\begin{aligned}
J(u) & =\frac{a}{2}\|u\|^{2}+\frac{b}{4}\|u\|^{4}-\sum_{k=1}^{N} F(k, u(k)) \\
& \leq \frac{a}{2}\|u\|^{2}+\frac{b}{4}\|u\|^{4}-\sum_{k=1}^{N}\left(\frac{b}{4}\left(\mu_{2}+\varepsilon\right) u^{4}(k)-M_{\varepsilon}\right) \\
& \leq \frac{a}{2}\|u\|^{2}-\frac{b \varepsilon}{4}\|u\|_{4}^{4}+N M_{\varepsilon} \\
& \leq \frac{a}{2}\|u\|^{2}-\frac{b \varepsilon}{4 N \lambda_{N}^{2}}\|u\|^{4}+N M_{\varepsilon},
\end{aligned}
$$

and thus

$$
J(u) \rightarrow-\infty, \quad \text { as }\|u\| \rightarrow \infty,
$$

which means that $J$ is inverse coercive in $H$. Because $J$ is continuous, $J$ must have a maximum point $u_{2}$. By Lemma 2.3(ii), we can conclude that

$$
C_{q}\left(J, u_{2}\right)=\delta_{q, N} \mathbb{F}
$$


By (3.2), (3.4) and $m \neq N$, it is easy to see that $u_{2} \neq 0$. Therefore $J$ has at least a nontrivial critical point, which completes the proof.

Proof of Theorem 1.2 We have (3.2) from (1.2), $f_{0}=\lambda_{m}$ and $\left(\mathrm{F}_{0}\right)$.

(i) Let $G(k, t)=F(k, t)-\frac{b}{4} \mu_{1} t^{4}$, then $\lim _{|t| \rightarrow+\infty} \frac{G(k, t)}{|t|^{4}}=0$ by (1.3) and $f_{\infty}=\mu_{1}$. Meanwhile, combining $\left(\mathrm{F}_{\infty}^{1}\right)$, we can get $\lim _{|t| \rightarrow \infty} G(k, t)=-\infty[9,18]$. This means that $\lim _{\|u\| \rightarrow \infty} \sum_{k=1}^{N} G(k, u(k))=-\infty$. Then

$$
\begin{aligned}
J(u) & =\frac{a}{2}\|u\|^{2}+\frac{b}{4}\|u\|^{4}-\sum_{k=1}^{N} F(k, u(k)) \\
& \geq \frac{a}{2}\|u\|^{2}+\frac{b}{4}\|u\|^{4}-\frac{b}{4} \mu_{1}\|u\|_{4}^{4}-\sum_{k=1}^{N} G(k, u(k)) \\
& \geq \frac{a}{2}\|u\|^{2}-\sum_{k=1}^{N} G(k, u(k)),
\end{aligned}
$$

obviously,

$$
J(u) \rightarrow \infty \quad \text { as }\|u\| \rightarrow \infty .
$$

Hence $J$ is coercive. Since $J$ is continuous, $J$ must have a minimum point $u_{1}$. Thus we obtain

$$
C_{q}\left(J, u_{1}\right)=\delta_{q, 0} \mathbb{F}
$$

From (3.2), (3.5) and $m \neq 0$, we can see that $u_{1} \neq 0$. So, $J$ has at least a nontrivial critical point.

(ii) One concludes that there exists $M>0$ such that $F(k, t) \geq c t^{2}+\frac{b}{4} \mu_{2} t^{4}-M$ for all $t \in \mathbb{R}$ by (1.3), $f_{\infty}=\mu_{2},\left(\mathrm{~F}_{\infty}^{2}\right)$ and the continuity of $F$. Hence, we infer from (2.1) and (2.4) that

$$
\begin{aligned}
J(u) & =\frac{a}{2}\|u\|^{2}+\frac{b}{4}\|u\|^{4}-\sum_{k=1}^{N} F(k, u(k)) \\
& \leq \frac{a}{2}\|u\|^{2}+\frac{b}{4}\|u\|^{4}-\sum_{k=1}^{N}\left(\frac{b}{4} \mu_{2} u^{4}(k)+c u^{2}(k)-M\right) \\
& =\frac{a}{2}\|u\|^{2}+\frac{b}{4}\|u\|^{4}-\frac{b}{4} \mu_{2}\|u\|_{4}^{4}-c\|u\|_{2}^{2}+N M \\
& \leq\left(\frac{a}{2}-\frac{c}{\lambda_{N}}\right)\|u\|^{2}+N M
\end{aligned}
$$

Note that $c \in\left(\frac{a}{2} \lambda_{N},+\infty\right)$, which gives $J(u) \rightarrow-\infty$ as $\|u\| \rightarrow+\infty$. This means that $J$ is inverse coercive in $H$. Due to the continuity of $J, J$ must have a maximum point $u_{2}$. Therefore we have

$$
C_{q}\left(J, u_{2}\right)=\delta_{q, N} \mathbb{F}
$$

From (3.2), (3.6) and $m \neq N$, we get $u_{2} \neq 0$. So, $J$ has at least a nontrivial critical point. The proof is completed. 
Proof of Theorem 1.3 It follows from (1.2), $f_{0} \neq \lambda_{i}, i \in \mathbb{Z}[1, N]$, and $J^{\prime \prime}(0)=a I_{N}-A^{-1} f_{t}^{\prime}(k, 0)$, $k \in \mathbb{Z}[1, N]$ that $u=0$ is a non-degenerate critical point of $J$ with the Morse index $\mu_{0}$. By Lemma 2.3(iii), we know that

$$
C_{q}(J, 0)=\delta_{q, \mu_{0}} \mathbb{F}
$$

(i) Equation (1.3), $f_{\infty}=\mu_{1}$ and $\left(\mathrm{F}_{\infty}^{1}\right)$ mean that (3.5) holds. Thus, by (3.5), (3.7) and $\mu_{0} \neq 0$, it is easily seen that $u_{1} \neq 0$, which implies that $J$ has at least a nontrivial critical point.

(ii) It follows from (1.3), $f_{\infty}=\mu_{2}$ and ( $\left.\mathrm{F}_{\infty}^{2}\right)$ that (3.6) holds. Therefore by (3.6), (3.7) and $\mu_{0} \neq N$, we have $u_{2} \neq 0$. This shows that $J$ admits at least a nontrivial critical point. Then the conclusion holds.

Proof of Theorem 1.4 It is easy to verify from (1.2) and $f_{0} \neq \lambda_{i}, i \in \mathbb{Z}[1, N]$ that (3.7) holds.

(i) Equation (1.3) and $f_{\infty}<\mu_{1}$ show that (3.3) holds. Hence, by (3.3), (3.7) and $\mu_{0} \neq 0$, we have $u_{1} \neq 0$. This implies that $J$ has at least a nontrivial critical point.

(ii) We can deduce (3.4) from (1.3) and $f_{\infty}>\mu_{2}$. Therefore we can obtain $u_{2} \neq 0$ from (3.4), (3.7) and $\mu_{0} \neq N$. This implies that $J$ has at least a nontrivial critical point. This proof is completed.

\section{Competing interests}

The authors declare that they have no competing interests.

\section{Authors' contributions}

The authors have made this manuscript independently. The authors read and approved the final version.

\section{Acknowledgements}

We are grateful to the referees for their suggestions that helped improve the paper greatly. In addition, this work is supported by the Natural Science Foundation of Shanxi Province (No. 2012011004-3).

\section{Received: 25 May 2013 Accepted: 14 October 2013 Published: 08 Nov 2013}

\section{References}

1. Perera, K, Zhang, ZT: Nontrivial solutions of Kirchhoff type problems via the Yang index. J. Differ. Equ. 221, 246-255 (2006)

2. Sun, JJ, Tang, CL: Resonance problems for Kirchhoff type equations. Discrete Contin. Dyn. Syst. 5, 2139-2154 (2013)

3. Bin, HH, Yu, JS, Guo, ZM: Nontrivial periodic solutions for asymptotically linear resonant difference problem. J. Math. Anal. Appl. 322, 477-488 (2006)

4. Zhu, BS, Yu, JS: Multiple positive solutions for resonant difference equations. Math. Comput. Model. 49, 1928-1936 (2009)

5. Zheng, B, Zhang, QQ: Existence and multiplicity of solutions of second-order difference boundary value problems. Acta Appl. Math. 110, 131-152 (2010)

6. Zheng, B, Xiao, HF: Existence of multiple solutions of a second-order difference boundary value problem. Int. J. Math. Math. Sci. 2010, Article ID 907453 (2010)

7. Liu, JS, Wang, SL, Zhang, JM: Multiple solutions for boundary value problems of second-order difference equations with resonance. J. Math. Anal. Appl. 374, 187-196 (2011)

8. Zhang, XS, Wang, D: Multiple periodic solutions for difference equations with double resonance at infinity. Adv. Differ. Equ. 2011, Article ID 806458 (2011)

9. Liu, JS, Wang, SL, Zhang, JM, Zhang, FW: Nontrivial solutions for resonant difference systems via computations of the critical groups. J. Math. Anal. Appl. 385, 60-71 (2012)

10. Liu, JS, Wang, SL, Zhang, JM, Zhang, FW: Nontrivial solutions for discrete boundary value problems with multiple resonance via computations of the critical groups. Nonlinear Anal. 75, 3809-3820 (2012)

11. Wang, SL, Liu, JS: Nontrivial solutions of a second order difference systems with multiple resonance. Appl. Math Comput. 218, 9342-9352 (2012)

12. Wang, SL, Liu, JS, Zhang, JM, Zhang, FW: Existence of non-trivial solutions for resonant difference equations. J. Differ. Equ. Appl. 19, 209-222 (2013)

13. Kirchhoff, G: Mechanik. Teubner, Leipzig (1883)

14. Lions, JL: On some questions in boundary value problems of mathematical physics. In: Contemporary Developments in Continuum Mechanics and Partial Differential Equations. North-Holland Math. Stud., vol. 30, pp. 284-346. North-Holland, Amsterdam (1978) 
15. Zhang, ZT, Perera, K: Sign-changing solutions of Kirchhoff type problems via invariant sets of descent flow. J. Math. Anal. Appl. 317, 456-463 (2006)

16. Li, YH, Li, FY, Shi, JP: Existence of a positive solution to Kirchhoff type problems without compactness conditions. J. Differ. Equ. 253, 2285-2294 (2012)

17. Xu, JX, Zhang, FB: Multiplicity and concentration of positive solutions for a Kirchhoff type problem with critical growth. J. Differ. Equ. 253, 2314-2351 (2012)

18. Yang, Y, Zhang, JH: Nontrivial solutions of a class of nonlocal problems via local linking theory. Appl. Math. Lett. 23, 377-380 (2010)

19. Deimling, K: Nonlinear Functional Analysis. Springer, Berlin (1985)

20. Chang, KC: Infinite Dimensional Morse Theory and Multiple Solutions Problem. Birkhäuser Boston, Boston (1993)

21. Liu, JQ: A Morse index of a saddle point. J. Syst. Sci. Math. Sci. 2, 32-39 (1989)

22. Su, JB: Semilinear elliptic boundary value problems with double resonance between two consecutive eigenvalues. Nonlinear Anal. 48, 881-895 (2002)

10.1186/1687-1847-2013-308

Cite this article as: Yang and Liu: Nontrivial solutions for discrete Kirchhoff-type problems with resonance via critical groups. Advances in Difference Equations 2013, 2013:308

\section{Submit your manuscript to a SpringerOpen ${ }^{\circ}$ journal and benefit from:}

- Convenient online submission

- Rigorous peer review

- Immediate publication on acceptance

Open access: articles freely available online

- High visibility within the field

- Retaining the copyright to your article 